DESY 98-065

May 1998

\title{
Chiral Fermions on the Lattice*)
}

\author{
V. Bornyakov ${ }^{1}$, G. Schierholz ${ }^{2,3}$ and A. Thimm ${ }^{2,4}$ \\ ${ }^{1}$ Institute for High Energy Physics IHEP, RU-142284 Protvino, Russia \\ ${ }^{2}$ Deutsches Elektronen-Synchrotron DESY, D-15735 Zeuthen, Germany \\ ${ }^{3}$ Deutsches Elektronen Synchrotron DESY, D-22603 Hamburg, Germany \\ ${ }^{4}$ Institut für Theoretische Physik, Freie Universität Berlin, D-14195 Berlin, \\ Germany
}

\begin{abstract}
A recently proposed method for regularizing chiral gauge theories non-perturbatively is discussed in detail. The result is an effective action which can be computed from the lattice gauge field, and which is suited for numerical simulations.
\end{abstract}

\section{§1. Introduction}

Formulating chiral gauge theories on the lattice is one of the great challenges in particle physics. The solution of this problem would lead to a new, qualitative and quantitative understanding of these theories.

To pay tribute to the topological nature of the problem, it has been suggested ${ }^{1)}$ to discretize the gauge fields only and consider the fermions in the continuum. By this we mean the following. One starts from a lattice with lattice spacing $a$. We call this lattice the original lattice. This is also the lattice on which the simulations of the gauge fields will be done. Then one constructs a finer lattice with lattice spacing $a_{f}$. On this lattice one places the fermions. We shall assume Wilson fermions to remove the doublers. Before one can state the action on the fine lattice, one has to extrapolate the gauge fields to the interior of the original lattice. A suitable extrapolation was given in ref. 2. The effective fermionic action is then derived in a two-step procedure. In the first step one computes the lattice effective action in the limit $a_{f} \rightarrow 0$, while keeping $a$ fixed. This action is generally not invariant under chiral gauge transformations. Chiral gauge invariance can, however, be restored by adding a few local bosonic counterterms to the action. The second step then is to determine the counterterms. This can be done perturbatively. The resulting action is a non-local function of the gauge fields on the original lattice. One might think that the action will be too complicated to be of any use. But we shall see that this is not the case. On the contrary: a simple effective action emerges which lends itself to numerical simulations. For similar ideas see ref. 3.

In this talk we shall test our idea in the chiral Schwinger model. The Schwinger model is particularly well suited for this task because a lot is known analytically. The talk is organized as follows. In sec. 2 we present some selected analytic results.

*) Talk given by G. Schierholz at Yukawa International Seminar on Non-Perturbative QCD: Structure of the QCD Vacuum (YKIS97), Kyoto, December 1997 
In sec. 3 we derive the effective fermionic action including the counterterms. In sec. 4 we then show that the resulting action is gauge invariant, and we compare numerical and analytic results. Finally, in sec. 5 we conlude.

\section{$\S 2 . \quad$ Analytic Results}

We shall mainly be concerned with the fermionic action. In the vector Schwinger model it reads

$$
S=\sum_{\alpha} \int \mathrm{d}^{2} x \bar{\psi}_{\alpha}(x) \not D\left(e_{\alpha} A\right) \psi_{\alpha}(x),
$$

where the sum is over fermion flavors, $e_{\alpha}$ is the charge of the fermion in units of $e$, and

$$
D_{\mu}(A)=\partial_{\mu}+\mathrm{i} A_{\mu}
$$

Note that $e_{\alpha}$ is dimensionless, but $e$ and $A_{\mu}$ are dimensionful. The model has a topological charge

$$
Q=\frac{1}{4 \pi} \int \mathrm{d}^{2} x \epsilon_{\mu \nu} F_{\mu \nu}(x) \in \mathbb{Z}
$$

The Schwinger model has been solved analytically in $\mathbb{R}^{24)}$, on the sphere $\mathbb{S}^{2}$ ) , as well as on the torus $\mathbb{T}^{26)-8)}$.

In the chiral Schwinger model the fermionic action reads

$$
S=\sum_{\alpha} \int \mathrm{d}^{2} x \bar{\psi}_{\alpha}(x) \not D^{\epsilon_{\alpha}}\left(e_{\alpha} A\right) \psi_{\alpha}(x),
$$

where $\epsilon_{\alpha}= \pm 1$ is the chirality, and

$$
D_{\mu}^{\epsilon_{\alpha}}(A)=\partial_{\mu}+\mathrm{i} A_{\mu} P_{\epsilon_{\alpha}}
$$

with

$$
P_{\epsilon_{\alpha}}=\frac{1}{2}\left(1+\epsilon_{\alpha} \gamma_{5}\right), \gamma_{5}=\mathrm{i} \gamma_{1} \gamma_{2}=\left(\begin{array}{rr}
-1 & 0 \\
0 & 1
\end{array}\right) .
$$

In eq. $(2 \cdot 4)$ the sum is over flavors and chiralities. The action $(2 \cdot 4)$ is invariant under chiral gauge transformations $g^{\alpha}=\exp \left(\mathrm{i} e_{\alpha} h P_{\epsilon_{\alpha}}\right)$ :

$$
\begin{aligned}
& \psi_{\alpha} \rightarrow \psi_{\alpha}^{g}=g^{\alpha} \psi_{\alpha}, \\
& \bar{\psi}_{\alpha} \rightarrow \bar{\psi}_{\alpha}^{g}=\bar{\psi}_{\alpha} g^{\alpha}, \\
& A_{\mu} \rightarrow A_{\mu}^{g}=A_{\mu}-\partial_{\mu} h .
\end{aligned}
$$

The anomaly cancelling condition is

$$
\sum_{\alpha} \epsilon_{\alpha} e_{\alpha}^{2}=0
$$

The effective fermionic action is defined by

$$
\exp (-W)=\int \mathcal{D} \bar{\psi} \mathcal{D} \psi \exp (-S)
$$


In $\mathbb{R}^{2}$, in a background of trivial topology $Q=0$, the effective action is known analytically both for the vector and the chiral model ${ }^{4), 9), 10)}$. After subtracting the divergent contribution arising from the free fermions, we obtain

$$
W(A)-W_{0}=\sum_{\alpha} \frac{e_{\alpha}^{2}}{8 \pi} \int \mathrm{d}^{2} x A_{\mu}(x)\left[a \delta_{\mu \nu}-\left(\partial_{\mu}+\mathrm{i} \epsilon_{\alpha} \widetilde{\partial}_{\mu}\right) \frac{1}{\square}\left(\partial_{\nu}+\mathrm{i} \epsilon_{\alpha} \widetilde{\partial}_{\nu}\right)\right] A_{\nu}(x),
$$

where $W_{0}=W(0)$,

$$
\widetilde{\partial}_{\mu}=\epsilon_{\mu \nu} \partial_{\nu}
$$

and $a$ (not to be confused with the lattice spacing $a$ ) reflects a regularization ambiguity 9$), 11)$. The one-loop perturbative result corresponds to $(2 \cdot 10)$ with $a=1$. For $a=1$ the effective action is invariant under chiral gauge transformations $(2 \cdot 7)$. It follows that

$$
\operatorname{Re} W(A)=\frac{1}{2}\left(W_{V}(A)+W_{0}\right)
$$

where $W_{V}$ is the effective action of the corresponding vector model. Moreover, we find

$$
\operatorname{Im} W(A)=0
$$

in the anomaly-free model. For an extension of the results to non-trivial topology see ref. 12 .

On the $T_{1} \times T_{2}$ torus the gauge field can be decomposed according to

$$
A_{\mu}(x)=\frac{2 \pi}{T_{\mu}} t_{\mu}+\partial_{\mu} h(x)+\widetilde{\partial}_{\mu} f(x)+C_{\mu}^{Q}(x),
$$

where $t_{\mu}$ is the zero-momentum component of the gauge field, called toron, $\partial_{\mu} h$ represents the pure gauge degrees of freedom, and $\widetilde{\partial}_{\mu} f$ and $C_{\mu}^{Q}$ are the proper dynamical components of zero and non-zero topological charge, respectively. Note, however, that this decomposition is not unique with respect to large gauge transformations ${ }^{8)}$. Let us now consider the sector of zero topological charge $Q=0$. For the gauge field we then may write

$$
A_{\mu}(x)=a_{\mu}+b_{\mu}(x),
$$

where $a_{\mu}=\left(2 \pi / T_{\mu}\right) t_{\mu}$ and $b_{\mu}(x)=\widetilde{\partial}_{\mu} f(x)+\partial_{\mu} h(x)$. We will show now that the effective action factorizes:

$$
W(A)=W(a)+W(b)
$$

We define

$$
A_{\mu}^{\tau}(x)=\tau A_{\mu}(x),
$$

with $0 \leq \tau \leq 1$. The effective action is then given by ${ }^{13)}$

$$
\begin{aligned}
W(A)-W_{0} & =-\frac{1}{2} \sum_{\alpha} \int_{0}^{1} \mathrm{~d} \tau \operatorname{Tr}\left(\frac{\mathrm{d}}{\mathrm{d} \tau} \not D^{\epsilon_{\alpha}}\left(e_{\alpha} A^{\tau}\right)\right) \not D\left(e_{\alpha} A^{\tau}\right)^{-1} \\
& =-\frac{\mathrm{i}}{2} \sum_{\alpha} e_{\alpha} \int_{0}^{1} \mathrm{~d} \tau \operatorname{Tr} \not A P_{\epsilon_{\alpha}} \not D\left(e_{\alpha} A^{\tau}\right)^{-1} .
\end{aligned}
$$


The particular feature of this expression is that it involves the covariant Dirac operator of the vector model only. To make the expression well defined, we use point splitting regularization:

$$
\begin{aligned}
W(A)-W_{0}= & -\lim _{\epsilon \rightarrow 0} \frac{\mathrm{i}}{4} \sum_{\alpha}\left\{e_{\alpha} \int_{0}^{1} \mathrm{~d} \tau \int \mathrm{d}^{2} x \not A(x) P_{\epsilon_{\alpha}} G\left(e_{\alpha} A^{\tau} \mid x, x+\epsilon\right)\right. \\
& \left.\times \exp \left(\mathrm{i} \int_{x+\epsilon}^{x} \mathrm{~d} z_{\mu} A_{\mu}^{\tau}(z)\right)+(\epsilon \leftrightarrow-\epsilon)\right\}
\end{aligned}
$$

where the fermion propagator $G(A \mid x, y)$ is a solution of the equation

$$
\not D(A) G(A \mid x, y)=\delta(x-y)
$$

with $\delta(x-y)$ being the torus $\delta$ function. If $G(A \mid x, 0)$ is a solution of $(2 \cdot 21)$ with periodic boundary conditions,

$$
G\left(A \mid x+m T_{1}+n T_{2}, 0\right)=G(A \mid x, 0)
$$

then

$$
G^{c}(A \mid x, 0)=\exp (\mathrm{i} c x) G(A+c \mid x, 0)
$$

is a solution with boundary conditions

$$
G^{c}\left(A \mid x+m T_{1}+n T_{2}, 0\right)=\exp \left(\mathrm{i} m c_{1} T_{1}+\mathrm{i} n c_{2} T_{2}\right) G^{c}(A \mid x, 0) .
$$

For $c_{\mu}=\pi / T_{\mu}$ this corresponds to anti-periodic boundary conditions. The propagator can be written

$$
G(A \mid x, y)=\exp \left(\mathrm{i} h(x)+\gamma_{5} f(x)\right) G(a \mid x, y) \exp \left(-\mathrm{i} h(y)+\gamma_{5} f(y)\right) .
$$

At short distances $|x-y|$ we have $^{8)}$

$$
G(a \mid x, y)=\exp (-\mathrm{i} a(x-y))\left(G_{0}(x, y)-\frac{1}{2 \pi} K(a)\right)+O(|x-y|),
$$

with $G_{0}(x, y)=G(0 \mid x, y)$ and

$$
K(a)=\left(\begin{array}{cc}
0 & -\mathrm{i} a_{1}+\frac{1}{T_{1}} \frac{\Theta_{1}^{\prime}\left(t_{-}\right)}{\Theta_{1}\left(t_{-}\right)} \\
-\mathrm{i} a_{1}+\frac{1}{T_{1}} \frac{\Theta_{1}^{\prime}\left(t_{+}\right)}{\Theta_{1}\left(t_{+}\right)} & 0
\end{array}\right),
$$

where $\Theta_{1}(x) \equiv \Theta_{1}\left(x \mid \mathrm{i} T_{2} / T_{1}\right)$ is the Jacobi function and $t_{ \pm}=\left(T_{2} / 2 \pi\right)\left(a_{2} \pm \mathrm{i} a_{1}\right)$. From eqs. $(2 \cdot 25)$ and $(2 \cdot 26)$ we see already that the effective action factorizes. It is straightforward now to evaluate eq. $(2 \cdot 20)$. Using standard techniques we obtain for general boundary conditions

$$
\begin{aligned}
W(A)-W_{0} & =\sum_{\alpha}\left\{\frac{T_{1} T_{2}}{4 \pi} e_{\alpha} a_{1}\left(e_{\alpha} a_{1}+2 c_{1}\right)-\ln \left|\Theta_{1}\left(e_{\alpha} t_{+}+c_{+}\right)\right|+\ln \left|\Theta_{1}\left(c_{+}\right)\right|\right. \\
& \left.-\mathrm{i} \epsilon_{\alpha}\left[\frac{T_{1} T_{2}}{4 \pi} e_{\alpha} a_{2}\left(e_{\alpha} a_{1}+2 c_{1}\right)+\arg \Theta_{1}\left(e_{\alpha} t_{+}+c_{+}\right)-\arg \Theta_{1}\left(c_{+}\right)\right]\right\} \\
& +\sum_{\alpha} \frac{e_{\alpha}^{2}}{8 \pi} \int \mathrm{d}^{2} x b_{\mu}\left[\delta_{\mu \nu}-\left(\partial_{\mu}+\mathrm{i} \epsilon_{\alpha} \widetilde{\partial}_{\mu}\right) \frac{1}{\square}\left(\partial_{\nu}+\mathrm{i} \epsilon_{\alpha} \widetilde{\partial}_{\nu}\right)\right] b_{\nu},
\end{aligned}
$$


where $c_{+}=\left(T_{2} / 2 \pi\right)\left(c_{2}+\mathrm{i} c_{1}\right)$. This proves eq. $(2 \cdot 16)$. It follows that

$$
\operatorname{Re} W(A)=\frac{1}{2}\left(W_{V}(A)+W_{0}\right)
$$

as in $\mathbb{R}^{2}$. In contrast to the previous result $(2 \cdot 13)$, the imaginary part of the effective action is no longer zero in the anomaly-free model. The reason for that is the toron field contribution ${ }^{14)}$ :

$$
\operatorname{Im} W(A)=-\sum_{\alpha} \epsilon_{\alpha}\left[\frac{T_{1} T_{2}}{4 \pi} e_{\alpha} a_{2}\left(e_{\alpha} a_{1}+2 c_{1}\right)+\arg \Theta_{1}\left(e_{\alpha} t_{+}+c_{+}\right)-\arg \Theta_{1}\left(c_{+}\right)\right] .
$$

A particular choice of $C_{\mu}^{Q}(x)$ is ${ }^{6), 8)}$

$$
C_{\mu}^{Q}(x)=-\frac{\pi Q}{T_{1} T_{2}} \epsilon_{\mu \nu} x_{\nu}
$$

The Dirac operator

$$
\not D^{Q}=\not \partial+\mathrm{i} e_{\alpha} Q^{Q}
$$

has $|Q|$ zero mode solutions:

$$
\not D^{Q} \chi_{l}^{Q}=0, l=1, \cdots,|Q| .
$$

Each eigenfunction has a definite chirality:

$$
\chi_{l}^{Q \dagger} \gamma_{5} \chi_{l}^{Q}=\left\{\begin{array}{cc}
+1 & Q>0 \\
-1 & Q<0
\end{array}\right.
$$

The number of zero modes $n_{+}\left(n_{-}\right)$with positive (negative) chirality is then given by

$$
\begin{aligned}
& n_{+}=Q \theta(Q), \\
& n_{-}=|Q| \theta(-Q),
\end{aligned}
$$

which is the index theorem (in two dimensions). Accordingly, the chiral Dirac operator $\not D^{Q \epsilon_{\alpha}}$ has $|Q|$ zero modes of chirality $\epsilon_{\alpha}$ if and only if $\epsilon_{\alpha}=\operatorname{sign}(Q)$. The eigenfunctions are the same as in the vector case. Later on we will use the expression

$$
C_{\mu}^{Q}(x)=2 \pi Q \epsilon_{\mu \nu} \partial_{\nu} G(x), x \in \dot{\mathbb{T}}^{2},
$$

where $\dot{T}^{2}$ is the torus with the point $x=0$ removed, and $G(x)$ is the inverse Laplacian on the torus satisfying the equation

$$
-\square G(x)=\delta(x)-\frac{1}{T_{1} T_{2}} .
$$

In analytic form ${ }^{8)}$

$$
G(x)=\frac{1}{2} \frac{x_{2}^{2}}{T_{1} T_{2}}-\frac{1}{2 \pi} \operatorname{Re} \ln \left(\frac{\Theta_{1}(z)}{\eta\left(\mathrm{i} T_{2} / T_{1}\right)}\right)
$$




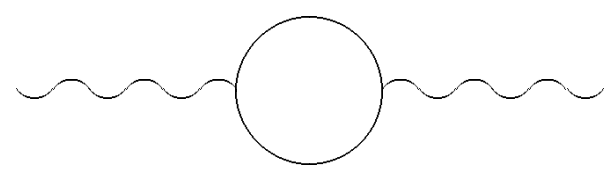

(a)

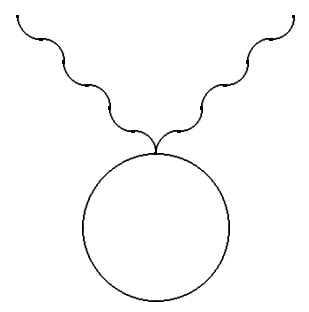

(b)

Fig. 1. Fermion loop diagrams contributing to the effective action.

where $z=\left(x_{1}+\mathrm{i} x_{2}\right) / T_{1}$ with $\Theta_{1}^{\prime}(0)=2 \pi \eta\left(\mathrm{i} T_{2} / T_{1}\right)^{3}$. The gauge field $(2 \cdot 36)$ is periodic, and it is related to the former expression $(2 \cdot 31)$ by the gauge transformation

$$
h(x)=Q \operatorname{Re} i \ln \left(\frac{\Theta_{1}(z)}{\eta\left(\mathrm{i} T_{2} / T_{1}\right)}\right)+\frac{\pi Q}{T_{1} T_{2}} x_{1} x_{2} .
$$

\section{§3. Effective Fermionic Action}

We shall first compute the lattice effective fermionic action and then determine the counterterms. We start from a $L_{1} \times L_{2}$ lattice with lattice spacing $a$. From this lattice we construct a fine $L_{1}^{f} \times L_{2}^{f}$ lattice with lattice spacing $a_{f}$. In practice $a_{f}=a / N, N \equiv L_{\mu}^{f} / L_{\mu} \in \mathbb{N}$. Let $n_{\mu}, 1 \leq n_{\mu} \leq L_{\mu}^{f}$, denote the points on the fine lattice. The action for one species of fermion with charge $e_{\alpha}$ and chirality $\epsilon_{\alpha}$ is given by

$$
\begin{aligned}
S_{\epsilon_{\alpha}} & =\frac{1}{2 a_{f}} \sum_{n, \mu}\left\{\overline { \psi } ( n ) \gamma _ { \mu } \left[\left(P_{-\epsilon_{\alpha}}+P_{\epsilon_{\alpha}}\left(U_{\mu}^{f}(n)\right)^{e_{\alpha}} \psi(n+\hat{\mu})\right.\right.\right. \\
& \left.-\left(P_{-\epsilon_{\alpha}}+P_{\epsilon_{\alpha}}\left(U_{\mu}^{f}(n-\hat{\mu})\right)^{e_{\alpha}^{\dagger}} \psi(n-\hat{\mu})\right]\right\} \\
& +S_{W \epsilon_{\alpha}}\left(U^{f}\right),
\end{aligned}
$$

where $\hat{\mu}$ is a unit vector in $\mu$-direction on the fine lattice, and $S_{W \epsilon_{\alpha}}\left(U^{f}\right)$ is the Wilson term. On the fine lattice the link variables are

$$
U_{\mu}^{f}(n) \equiv \exp \left(\mathrm{i} \theta_{\mu}^{f}(n)\right)=\exp \left(\mathrm{i} a_{f} \int_{n}^{n+\hat{\mu}} \mathrm{d} z_{\mu} A_{\mu}(z)\right),
$$

where $A_{\mu}$ is the continuum gauge field obtained by extrapolation. 
The lattice effective action is given by

$$
\exp \left(-W_{\epsilon_{\alpha}}\right)=\int \mathcal{D} \bar{\psi} \mathcal{D} \psi \exp \left(-S_{\epsilon_{\alpha}}\right)
$$

Due to the presence of the Wilson term, this action is not invariant under chiral gauge transformations. Classically the Wilson term vanishes to order $a_{f}$. However, in fermion loops as shown in fig. 1 , which receive contributions from loop momenta of the order of the cut-off $\approx \pi / a_{f}$, the Wilson term will in general give a finite contribution to the effective action. Because the effective action refers to classical background gauge fields, we need not consider diagrams with internal gauge boson lines. In the limit $a_{f} \rightarrow 0$ the non-gauge invariant contribution of the loop integrals is expected to contract to a multi-gauge boson amplitude, so that chiral gauge invariance may be restored by adding a few local bosonic counterterms to the action. The advantage of this method, as opposed to the Rome approach ${ }^{16)}$, is that the counterterms can be computed perturbatively, and no (non-perturbative) fine-tuning of the coefficients is required. We write

$$
W_{\epsilon_{\alpha}}^{\Sigma}=W_{\epsilon_{\alpha}}+C,
$$

where $C$ denotes the counterterm. The task is then to determine $C$, so that

$$
\widehat{W}_{\epsilon_{\alpha}}=\lim _{a_{f} \rightarrow 0} W_{\epsilon_{\alpha}}^{\Sigma}
$$

is invariant under chiral gauge transformations in the anomaly-free model.

For the Wilson term one has several choices. We shall take

$$
\begin{aligned}
S_{W \epsilon_{\alpha}}\left(U^{f}\right)= & -\frac{r}{2} \sum_{n, \mu} \bar{\psi}(n) D_{\mu}^{\epsilon_{\alpha}+} D_{\mu}^{\epsilon_{\alpha}-} \psi(n) \\
= & \frac{r}{2 a_{f}} \sum_{n, \mu} \bar{\psi}(n)\left\{2 \psi(n)-\left[P_{-\epsilon_{\alpha}}+P_{\epsilon_{\alpha}}\left(U_{\mu}^{f}(n)\right)^{e_{\alpha}}\right] \psi(n+\hat{\mu})\right. \\
& \left.-\left[P_{-\epsilon_{\alpha}}+P_{\epsilon_{\alpha}}\left(U_{\mu}^{f}(n-\hat{\mu})\right)^{e_{\alpha} \dagger}\right] \psi(n-\hat{\mu})\right\},
\end{aligned}
$$

where the superscript $+(-)$ means forward (backward) derivative. This Wilson term has two important properties which, e.g., the ungauged and the gauged Wilson terms of the vector model do not have.

In order that the lattice theory is in the same universality class as the classically defined theory, it must obey the index theorem. The lattice fermionic action can be written in the matrix form

$$
\bar{\psi}\left(\not D^{\epsilon_{\alpha}}-\frac{r}{2} M^{\epsilon_{\alpha}}\right) \psi
$$

The Dirac operator $\not D^{\epsilon_{\alpha}}$ is a finite matrix now. Thus $\not D^{\epsilon_{\alpha}}$ and $\not D^{\epsilon_{\alpha} \dagger}$ have the same number of zero modes. It is furthermore easy to see that

$$
\not D^{\epsilon_{\alpha}^{\dagger}}=\not D^{-\epsilon_{\alpha}}
$$

This tells us that the chiral lattice Dirac operator has the same number of righthanded and left-handed zero modes, thus violating the index theorem $(2 \cdot 35)$. The 
situation is different if we include the Wilson term. A necessary requirement for the index theorem to be valid on the lattice is

$$
M^{\epsilon_{\alpha}^{\dagger}} \neq M^{-\epsilon_{\alpha}} .
$$

The Wilson term (3.6) fulfills this requirement, unlike the ungauged and the gauged Wilson term of the vector model. In ref. 17 we will show that the Wilson term $(3 \cdot 6)$ in fact fulfills the index theorem.

The Wilson term couples right- and left-handed fermions. In order that the theory is chiral, the ungauged fermion must decouple in the limit $a_{f} \rightarrow 0$. This is the case if the action is invariant under the transformations ${ }^{15)}$

$$
\begin{aligned}
& \psi_{-\epsilon_{\alpha}} \equiv P_{-\epsilon_{\alpha}} \psi \rightarrow \psi_{-\epsilon_{\alpha}}+\eta, \\
& \bar{\psi}_{-\epsilon_{\alpha}} \equiv \bar{\psi} P_{\epsilon_{\alpha}} \rightarrow \bar{\psi}_{-\epsilon_{\alpha}}+\bar{\eta},
\end{aligned}
$$

where $\eta, \bar{\eta}$ are constant Grassmann variables. We denote the fermion propagator corresponding to the action $(3 \cdot 1)$ in momentum space by $G_{\epsilon_{\alpha}}(p)$. If the action is invariant under the transformation $(3 \cdot 10)$, we find

$$
\begin{aligned}
G_{\epsilon_{\alpha}}^{-1}(p)= & \left(\frac{\mathrm{i}}{a_{f}} \gamma_{\mu} f_{\mu}^{\epsilon_{\alpha}}(p)+m^{\epsilon_{\alpha}}(p)\right) P_{\epsilon_{\alpha}} \\
& +\left(\frac{\mathrm{i}}{a_{f}} \gamma_{\mu} \sin \left(a_{f} p_{\mu}\right)+\frac{r}{a_{f}} \sum_{\mu}\left[1-\cos \left(a_{f} p_{\mu}\right)\right]\right) P_{-\epsilon_{\alpha}}
\end{aligned}
$$

and if the action is invariant under the transformation $(3 \cdot 11)$, we find

$$
\begin{aligned}
G_{\epsilon_{\alpha}}^{-1}(p)= & \left(\frac{\mathrm{i}}{a_{f}} \gamma_{\mu} f_{\mu}^{\epsilon_{\alpha}}(p)+\frac{r}{a_{f}} \sum_{\mu}\left[1-\cos \left(a_{f} p_{\mu}\right)\right]\right) P_{\epsilon_{\alpha}} \\
& +\left(\frac{\mathrm{i}}{a_{f}} \gamma_{\mu} \sin \left(a_{f} p_{\mu}\right)+m^{-\epsilon_{\alpha}}(p)\right) P_{-\epsilon_{\alpha}},
\end{aligned}
$$

where $f_{\mu}^{\epsilon_{\alpha}}$ and $m^{\epsilon_{\alpha}}(p), m^{-\epsilon_{\alpha}}(p)$ are left undetermined. If the action is invariant under both transformations, $(3 \cdot 10)$ and $(3 \cdot 11)$, as was originally considered in ref. 15 , then

$$
m^{\epsilon_{\alpha}}(p)=m^{-\epsilon_{\alpha}}(p)=\frac{r}{a_{f}} \sum_{\mu}\left[1-\cos \left(a_{f} p_{\mu}\right)\right] .
$$

Remember that the gauged (ungauged) fermion has chirality $\epsilon_{\alpha}\left(-\epsilon_{\alpha}\right)$. The Dirac part of the action $(3 \cdot 1)$ is invariant under both transformations $(3 \cdot 10)$ and $(3 \cdot 11)$. The Wilson term $(3 \cdot 6)$ is invariant under the transformation $(3 \cdot 10)$, but not under $(3 \cdot 11)$. From the propagator $(3 \cdot 12)$ we read off that in the limit $a_{f} \rightarrow 0$ the theory describes a free, massless fermion with chirality $-\epsilon_{\alpha}$, plus an interacting fermion with chirality $\epsilon_{\alpha}$. The interacting fermion will, in general, require a mass counterterm to become massless, while for the free fermion no tuning of the mass is necessary. This means that the ungauged fermion decouples. If the action is invariant under both transformations, then the interacting fermion will automatically be massless in the 
continuum limit, which follows from $(3 \cdot 14)$. The gauged Wilson term of the vector model, e.g., is not invariant under any of the two transformations.

We shall now compute the counterterm. In two dimensions the only diagrams one has to consider are those in fig. 1 . In the continuum they require (at most) a counterterm of the form

$$
c e_{\alpha}^{2} \int \mathrm{d}^{2} x A_{\mu}^{2}(x)
$$

To compute the coefficient $c$ we proceed as follows. The contribution of the diagrams in fig. 1 to the effective action has the form

$$
\sum_{\alpha} \frac{e_{\alpha}^{2}}{2} \int \mathrm{d}^{2} k A_{\mu}(k) \Pi_{\mu \nu}(k) A_{\nu}(-k)
$$

where $\Pi_{\mu \nu}(k)$ is the polarization tensor. The polarization tensor is given by

$$
\Pi_{\mu \nu}(k)=\left(\delta_{\mu \nu} A+\frac{\left(k_{\mu}+\mathrm{i} \epsilon_{\alpha} \widetilde{k}_{\mu}\right)\left(k_{\nu}+\mathrm{i} \epsilon_{\alpha} \widetilde{k}_{\nu}\right)}{k^{2}} B\right)+O\left(a_{f}\right)
$$

where $\widetilde{k}_{\mu}=\epsilon_{\mu \nu} k_{\nu}$. For the Wilson term (3.6) the coefficients are

$$
A=\int_{-\pi}^{\pi} \frac{\mathrm{d}^{2} p}{(2 \pi)^{2}}\left\{\frac{2 c_{1}^{2} s_{1}^{2}+2 r c_{1} s_{1}^{2} \hat{s}^{2}+r^{2} s_{1}^{2}\left(\hat{s}^{2}\right)^{2}-c_{1}^{2} s^{2}}{\left(s^{2}+\left(\hat{s}^{2}\right)^{2}\right)^{2}}+\frac{s_{1}^{2}-r c_{1} \hat{s}^{2}}{s^{2}+\left(\hat{s}^{2}\right)^{2}}\right\}
$$

and

$$
\begin{aligned}
B=\int_{-\pi}^{\pi} \frac{\mathrm{d}^{2} p}{(2 \pi)^{2}}\{ & \frac{2 c_{1}^{2} s_{1}^{2}+4 r c_{1} s_{1}^{2} \hat{s}^{2}-r^{2} s_{1}^{2}\left(s^{2}-\left(\hat{s}^{2}\right)^{2}\right)-c_{1}^{2}\left(s^{2}+\left(\hat{s}^{2}\right)^{2}\right)}{\left(s^{2}+\left(\hat{s}^{2}\right)^{2}\right)^{2}} \\
& \left.+\frac{s_{1}^{2}-r c_{1} \hat{s}^{2}}{s^{2}+\left(\hat{s}^{2}\right)^{2}}\right\}
\end{aligned}
$$

where $c_{\mu}=\cos p_{\mu}, s_{\mu}=\sin p_{\mu}$ and $\hat{s}^{2}=2 r \sum_{\lambda} \sin ^{2}\left(p_{\lambda} / 2\right)$. The integral in (3.19) can be done analytically, giving

$$
B=\frac{1}{4 \pi}
$$

for all $r>0$. For $r=0$ the result is $B=1 / \pi$, which is a factor of four larger, as it should be, because we have four identical flavors in this case. For $(3 \cdot 18)$ and $r=1$ we obtain numerically

$$
A=0.199006 .
$$

The effective action, including the counterterm, is gauge invariant if $A+2 c=B$. Thus we find for the coefficient of the counterterm

$$
c=\frac{1}{2}(B-A)=-0.059714 \text {. }
$$




\section{$\S 4$. Numerical Test}

Because of lack of space we shall confine our tests to a few topics. Most important is the test of chiral gauge invariance of the effective action $(3 \cdot 5)$. If successful, it would mean that chiral gauge theories exist at the non-perturbative, constructive level. Among the analytic results that need to be tested are factorization of the effective action and eq. $(2 \cdot 30)$. These results are particularly important for numerical simulations of the theory.

Before we begin our tests, let us briefly mention the extrapolation we are using ${ }^{2}$. We start from the link variables on the original lattice,

$$
U_{\mu}(s) \equiv \exp \left(\mathrm{i} \theta_{\mu}(s)\right),-\pi<\theta_{\mu}(s) \leq \pi,
$$

where $s_{\mu} \in \mathbb{Z}$ are the lattice points on the original lattice. We define the plaquette variables

$$
P(s)=U_{1}(s) U_{2}(s+\hat{1}) U_{1}(s+\hat{2})^{\dagger} U_{2}(s)^{\dagger} \equiv \exp (\mathrm{i} \omega(s)),-\pi<\omega(s) \leq \pi .
$$

We then have

$$
\partial_{1}^{+} \theta_{2}(s)-\partial_{2}^{+} \theta_{1}(s)=\omega(s)-2 \pi n(s),
$$

with $n(s)=0, \pm 1$. The cases $\theta_{\mu}(s)=\pi, \omega(s)=\pi$ correspond to exceptional configurations, for which the topological charge is not defined. These configurations are of measure zero and will be excluded from our discussion. Following ref. 2 we then obtain for the continuum gauge field

$$
\begin{aligned}
& a A_{1}(x)=\theta_{1}(s)+\left[-\theta_{1}(s)+\theta_{1}(s+\hat{2})-2 \pi n(s)\right]\left(x_{2}-s_{2}\right)+2 \pi n(s) \theta\left(x_{2}-\bar{x}_{2}\right), \\
& a A_{2}(x)=\theta_{2}(s)+\left[-\theta_{2}(s)+\theta_{2}(s+\hat{1})\right]\left(x_{1}-s_{1}\right)+2 \pi n(s)\left(x_{1}-s_{1}\right) \delta\left(x_{2}-\bar{x}_{2}\right),
\end{aligned}
$$

where

$$
x \in c(s), c(s)=\left\{x \in \mathbb{T}^{2} \mid s_{\mu} \leq x_{\mu} \leq s_{\mu}+1\right\},
$$

and where $\bar{x}_{2}$ is implicitly defined by

$$
\theta_{1}(s)+\left[-\theta_{2}(s)+\theta_{2}(s+\hat{1})-\omega(s)\right]\left(\bar{x}_{2}-s_{2}\right)=-\pi n(s) .
$$

It follows that $-\pi \leq a A_{\mu}(x) \leq \pi$, except on the singular line $x_{2}=\bar{x}_{2}, s_{1} \leq x_{1} \leq$ $s_{1}+1$. The extrapolation is gauge covariant, and the parallel transporters derived from the continuum gauge field are consistent with those on the original lattice. In particular we have

$$
a^{2} F_{12}(x)=\omega(s), x \in c(s) .
$$

We first discuss the case of trivial topology. For compact lattice fields we have the decomposition

$$
\theta_{\mu}(s)=\frac{2 \pi}{L_{\mu}} t_{\mu}+\partial_{\mu}^{+} h(s)+\widetilde{\partial}_{\mu}^{-} f(s),
$$

where $f(s)$ is defined by $\square f(s)=\omega(s)$ with $\square=\partial_{\mu}^{+} \partial_{\mu}^{-}$. Unlike in the continuum, where $t_{\mu}$ was determined up to an integer, $t_{\mu}$ is non-invariant under a wider class of large gauge transformations. 


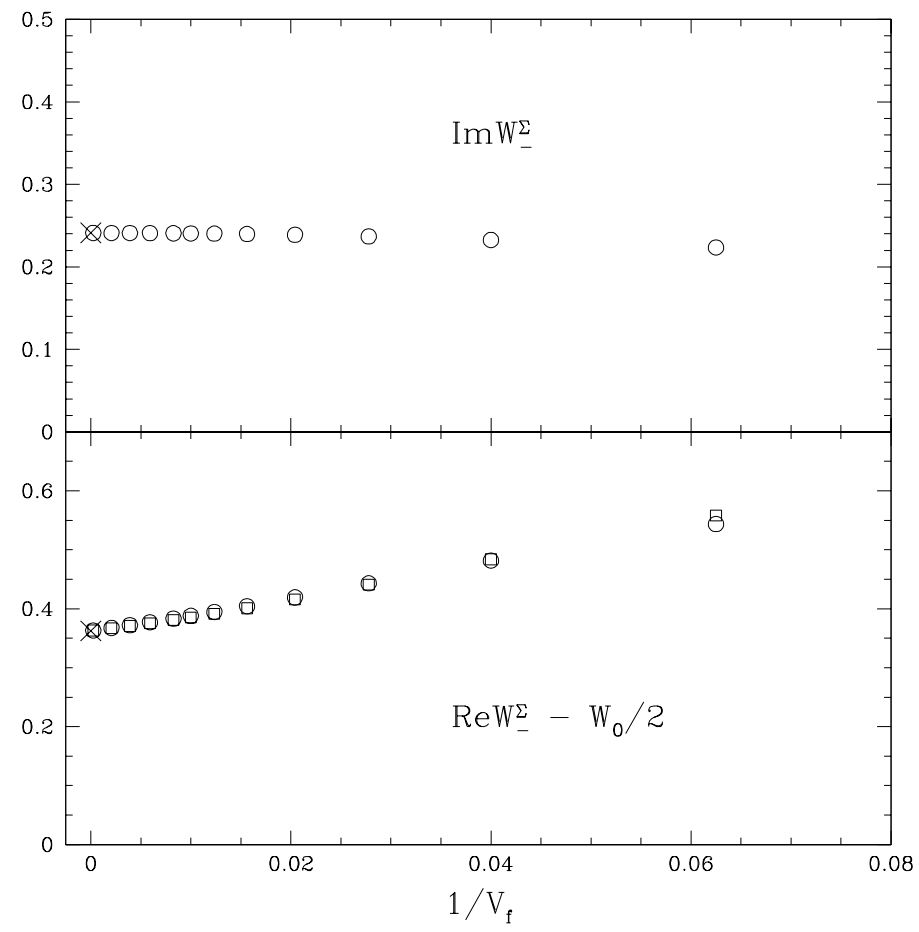

Fig. 2. The effective actions $\operatorname{Re} W_{-}^{\Sigma}-W_{0} / 2$ and $\operatorname{Im} W_{-}(\bigcirc)$ for a pure toron field configuration with $(t, \phi)=(0.5, \pi / 8)$ and $\left(e_{\alpha}, \epsilon_{\alpha}\right)=(1,-1)$ plotted against $1 / V_{f}$. Also shown is $(1 / 2) W_{V}$ $(\square)$. The numerical results are compared to the analytic results $(\times)$ given in $(2 \cdot 28),(2 \cdot 30)$.

All our calculations are for periodic boundary conditions for the gauge fields and anti-periodic boundary conditions for the fermions. We work on symmetric lattices with $L_{1}=L_{2}$. The lattice effective action is computed by means of the Lanczos algorithm ${ }^{18)}$. Note that the fermion matrix is not Hermitian in our case. The Lanczos vectors are re-orthogonalized after every iteration. Due to re-orthogonalization the CPU time grows with $V_{f}^{3}$, where $V_{f}=L_{1}^{f} \times L_{2}^{f}$, and the memory demand grows with $V_{f}^{2}$.

To begin with, let us consider pure toron fields. Writing

$$
t_{1}=t \cos \phi, t_{2}=t \sin \phi,
$$

we take

$$
t=0.5, \phi=\frac{\pi}{8}
$$

For this choice of $\phi$ the imaginary part of the effective action is close to its maximal value for any given value of $t$. As the anomaly is zero in this case, we may consider a single chiral fermion. We choose $e_{\alpha}=1$ and $\epsilon_{\alpha}=-1$. In fig. 2 we plot the effective action $(3 \cdot 4)$,

$$
W_{-}^{\Sigma}=W_{-}+C=W_{-}+c \sum_{n, \mu}\left(2-U_{\mu}^{f}(n)-U_{\mu}^{f}(n)^{\dagger}\right),
$$




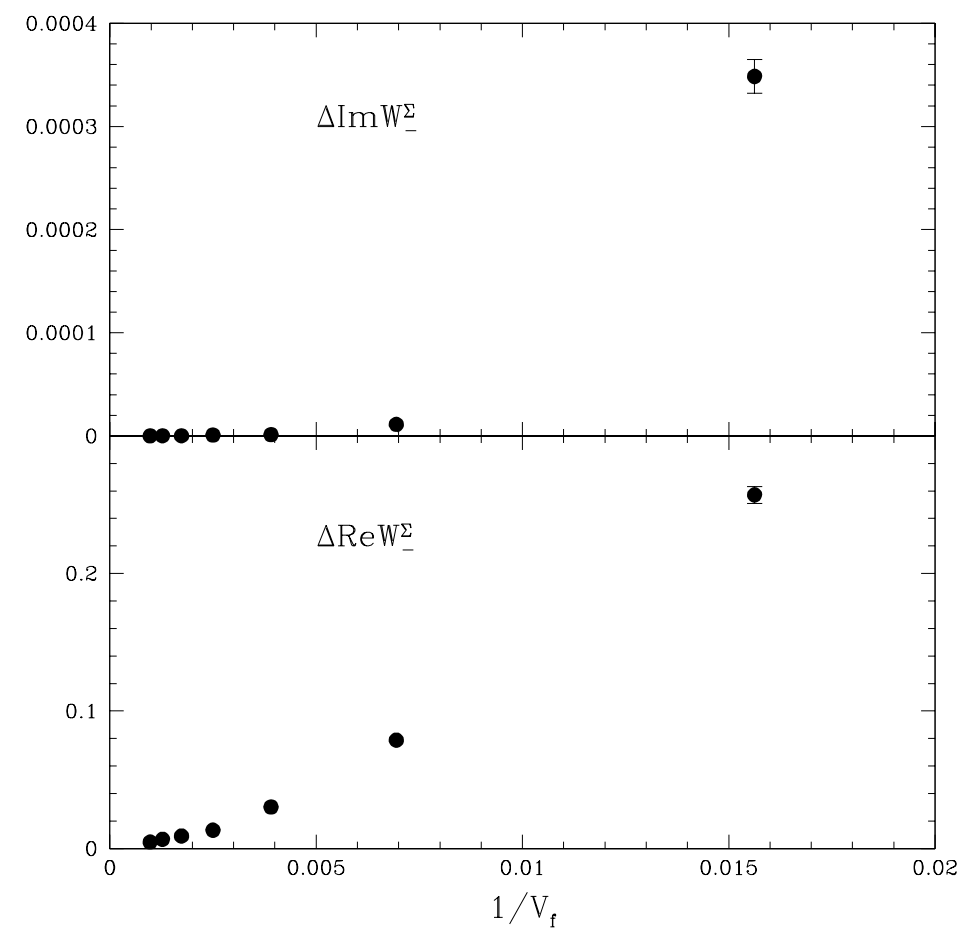

Fig. 3. The variance $\Delta \operatorname{Re} W_{-}^{\Sigma}$ and $\Delta \operatorname{Im} W_{-}$for the toron field configuration plotted against $1 / V_{f}$.

with $c$ being given by $(3 \cdot 22)$, as a function of $1 / V_{f}$. We see that the numerical results converge to the analytic values in the limit $a_{f} \rightarrow 0$. We furthermore see that $\operatorname{Re} W_{-}^{\Sigma} \rightarrow\left(W_{V}+W_{0}\right) / 2$.

Next we consider the effect of a small random lattice gauge transformation, described by $h(s)$, on the toron field configuration $(4 \cdot 10)$. To monitor the variation of the effective action under such transformations, we introduce the measure

$$
\Delta X=\frac{1}{N} \sum_{h}\left|X^{h}-X\right|,
$$

where the sum is over a set of $N$ gauge transformations, $X$ is the starting value, and $X^{h}$ is the result after the gauge transformation. If the quantity $X$ is gauge invariant, clearly $\Delta X=0$. In fig. 3 we plot $\Delta \operatorname{Re} W_{-}^{\Sigma}$ and $\Delta \operatorname{Im} W_{-}$as a function of $1 / V_{f}$. The number of gauge transformations $N$ varied between 20 and 100, depending on $V_{f}$. We see that both $\Delta \operatorname{Re} W_{-}^{\Sigma}$ and $\Delta \operatorname{Im} W_{-}$go to zero as expected in the limit $a_{f} \rightarrow 0$.

To check factorization $(2 \cdot 16)$ and the analytic results $(2 \cdot 28),(2 \cdot 30)$, one needs to consider more general gauge fields. We consider the continuum gauge field

$$
A_{\mu}(x)=c_{\mu} \cos \left(\frac{2 \pi k_{\mu} x_{\mu}}{T_{\mu}}\right)+\frac{2 \pi}{T_{\mu}} t_{\mu}
$$

corresponding to a plane wave plus toron solution. On the lattice this gives (cf. 


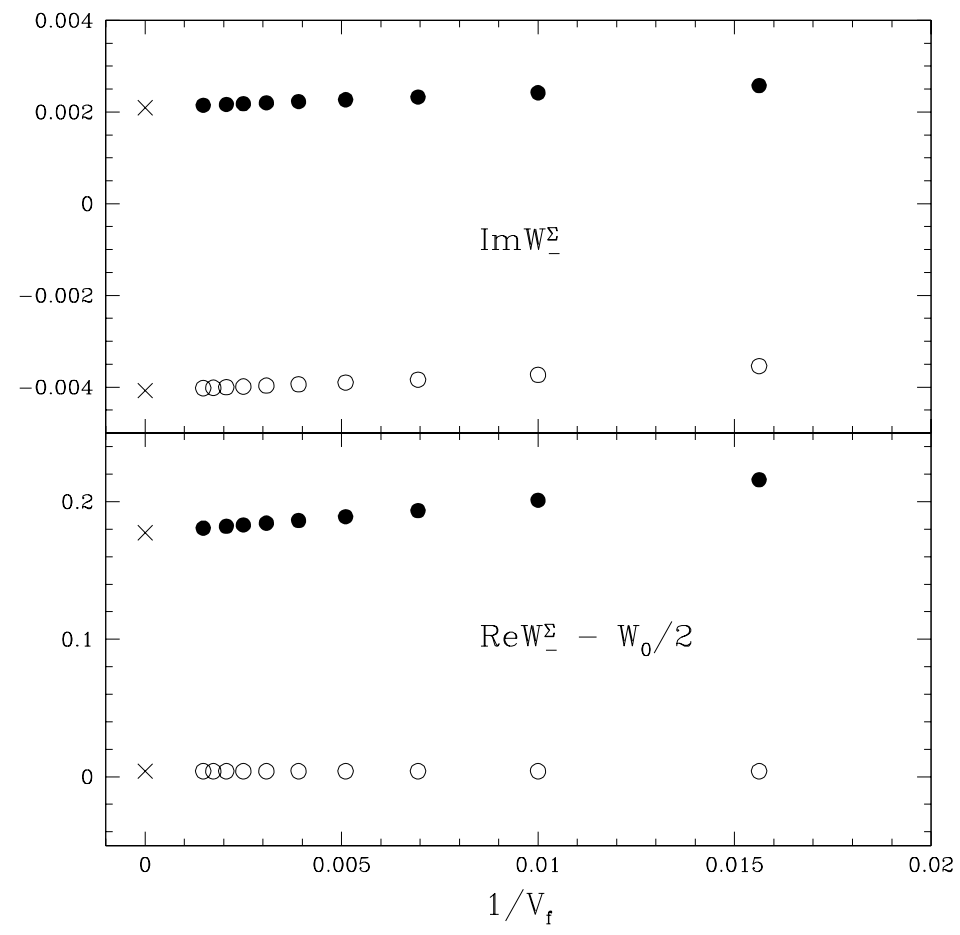

Fig. 4. The effective actions $\operatorname{Re} W_{-}^{\Sigma}-W_{0} / 2$ and $\operatorname{Im} W_{-}$for the configuration (4.14) with $t_{\mu}=0$

$(\bigcirc)$ and $(t, \phi)=(0.5, \pi / 400)(\bullet)$. The numerical results are compared to the analytic results

$(\times)$ given in $(2 \cdot 28),(2 \cdot 30)$.

eq. $(3 \cdot 2))$

$$
\theta_{\mu}^{f}(n)=a_{f} c_{\mu} \sin \left(\frac{\pi k_{\mu}}{L_{\mu}^{f}}\right)\left(\frac{\pi k_{\mu}}{L_{\mu}^{f}}\right)^{-1} \cos \left(\frac{2 \pi k_{\mu} n_{\mu}}{L_{\mu}^{f}}+\frac{\pi k_{\mu}}{L_{\mu}^{f}}\right)+\frac{2 \pi}{L_{\mu}^{f}} t_{\mu} .
$$

The virtue of this configuration is that it allows to compute the continuum effective action analytically. We choose $T_{1} c_{1}=T_{2} c_{2}=0.32$ and $k_{1}=1, k_{2}=0$. In fig. 4 we show $\operatorname{Re} W_{-}^{\Sigma}$ and $\operatorname{Im} W_{-}^{\Sigma}$ for $t_{\mu}=0$ and $t=0.5, \phi=\pi / 400$. The reason for choosing such a small value of $\phi$ was to make the plane wave and toron contribution about equal in magnitude. We see in both cases that the numerical results converge to the analytic values in the limit $a_{f} \rightarrow 0$. In particular this confirms factorization, and that the non-toron part of the effective action is given by the $\mathbb{R}^{2}$ result stated in eqs. $(2 \cdot 10)$ and $(2 \cdot 28)$.

We shall now consider a gauge field configuration generated by Monte-Carlo with the pure gauge field action at $\beta=1 / e^{2}=6.0$. We have chosen a configuration with no vortex-antivortex pairs. In this case the imaginary part of the effective action is not expected to be gauge invariant anymore for a single chiral fermion. But it is expected to be gauge invariant in the anomaly-free case. To test gauge invariance, we have computed $\Delta \operatorname{Re} W_{-}^{\Sigma}, \Delta \operatorname{Im} W_{-}^{\Sigma}$ as well as $\Delta \operatorname{Im} W_{a}^{\Sigma}$, where the subscript $a$ 


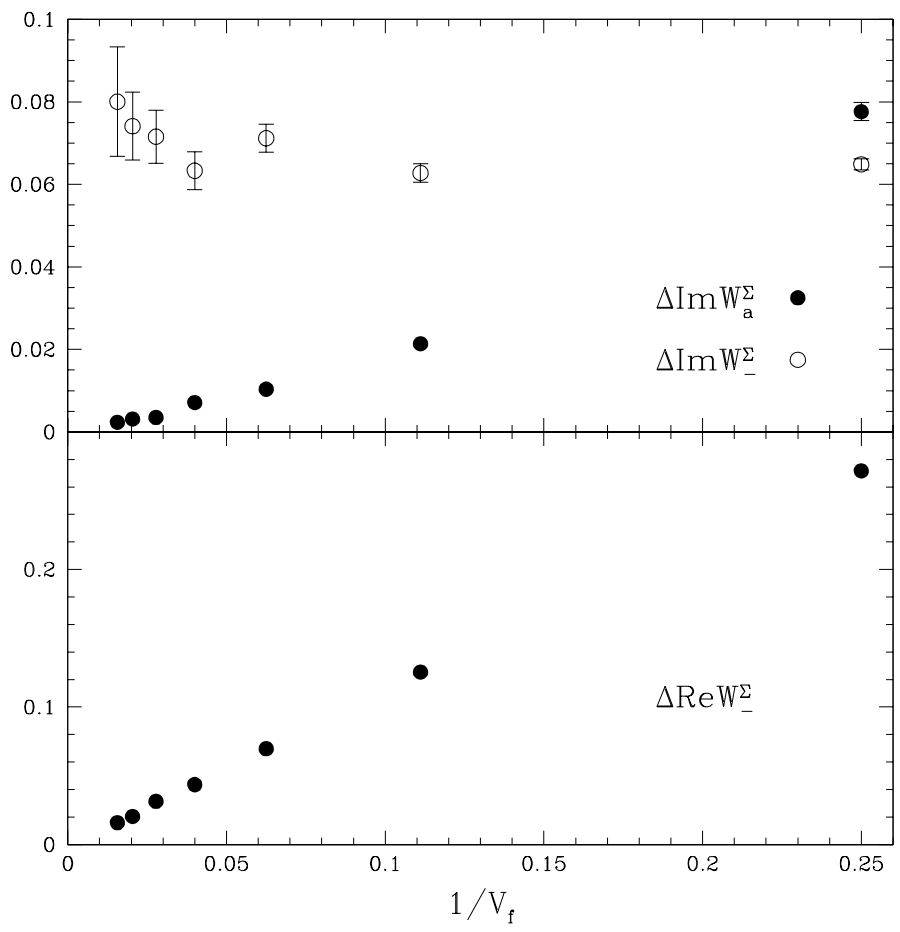

Fig. 5. The variance $\Delta \operatorname{Re} W_{-}^{\Sigma}$ and $\Delta \operatorname{Im} W_{a}(\bullet)$, together with $\Delta \operatorname{Im} W_{a}(\bigcirc)$, for a Monte Carlo generated configuration plotted against $1 / V_{f}$.

stands for the anomaly-free model with $\epsilon_{\alpha} e_{\alpha}=-1,-1,-1,-1,+2$. The result is shown in fig. 5. We see that $\Delta \operatorname{Re} W_{-}^{\Sigma}$ and $\Delta \operatorname{Im} W_{a}^{\Sigma}$ go to zero in the limit $a_{f} \rightarrow 0$, which means that gauge invariance is restored. On the other hand, we find that $\Delta \operatorname{Im} W_{-}^{\Sigma}$ does not vanish, which is a consequence of the presence of the anomaly.

Let us now turn to topologically non-trivial configurations. Here we restrict ourselves to the sector $|Q|=1$. For the lattice gauge field $C_{\mu}^{Q}$ we take (cf. $(2 \cdot 36)$ )

$$
C_{\mu}^{Q}(s)=2 \pi Q \epsilon_{\mu \nu} \partial_{\nu}^{-} G(s)
$$

where $G(s)$ is the inverse lattice Laplacian. We have checked that the action $(3 \cdot 1)$ satisfies the index theorem. We have furthermore verified that the effective action factorizes into a toron and a non-toron part, where both the real and imaginary part of the toron contribution are given by the analytic formulae $(2 \cdot 28)$ and $(2 \cdot 30)$. We have no space to show the results here. We have also checked gauge invariance. In fig. 6 we show $\Delta \operatorname{Re} W_{-}^{\Sigma}$ and $\Delta \operatorname{Im} W_{a}$ for the configuration (4.15) and a single random gauge transformation. We have divided out $\operatorname{Re} W_{-}^{\Sigma}$ because the action increases strongly with decreasing $1 / V_{f}$. We find that both $\operatorname{Re} W_{-}^{\Sigma}$ and $\operatorname{Im} W_{a}$ are gauge invariant in the limit $a_{f} \rightarrow 0$. 


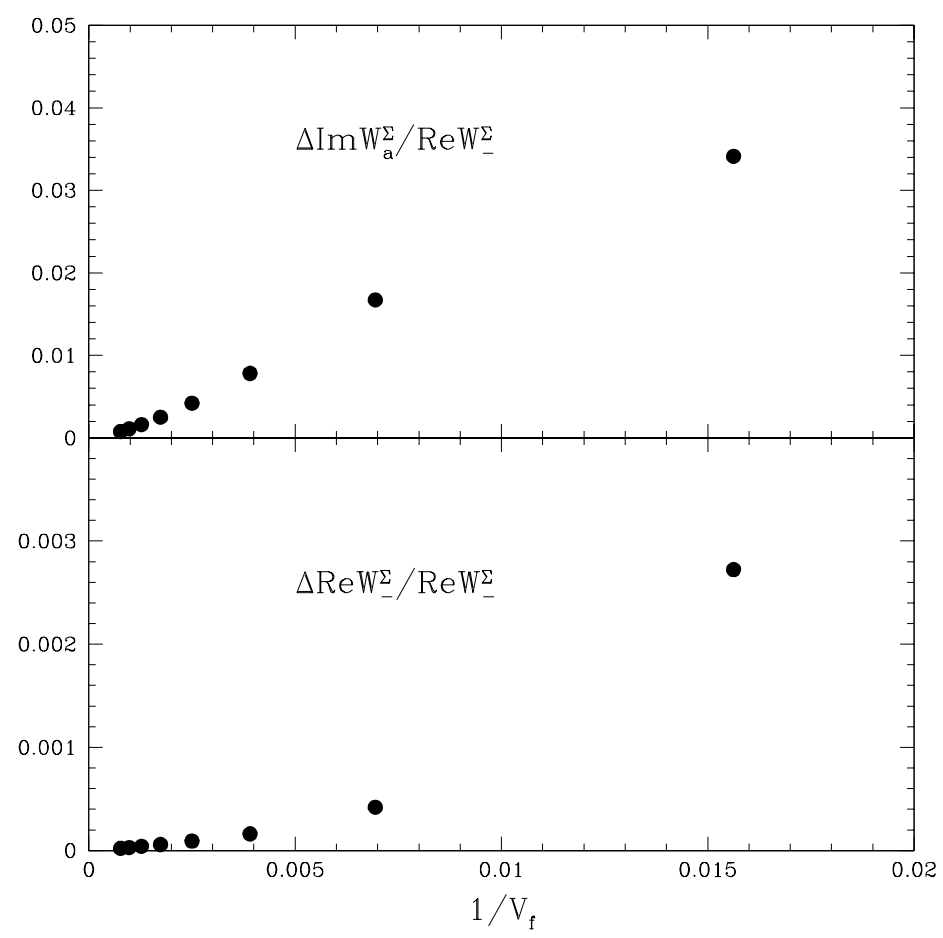

Fig. 6. The variance $\Delta \operatorname{Re} W_{-}^{\Sigma}$ and $\Delta \operatorname{Im} W_{a}$ for a $|Q|=1$ configuration plotted against $1 / V_{f}$.

\section{$\S 5$. Conclusions}

Due to space limitations we were only able to show some of our results. In particular, we had to omit the discussion of large gauge transformations. Some preliminary mention of this topic can be found in ref. 19. In general, the action is not invariant under such gauge transformations. The action of the transformed field will, however, diverge in the limit $a_{f} \rightarrow 0$, so that this configuration has zero weight in the partition function. This is not a shortcoming of our method. The same result is found in the continuum.

We may conclude that we have found a non-perturbative, gauge invariant formulation of chiral gauge theories. In the sector of trivial topology the real part of the effective action converges to $\left(W_{V}+W_{0}\right) / 2$ in the continuum limit, whereas the imaginary part becomes a function of the toron field only in the anomaly-free model. The effective action may then be written

$$
\widehat{W}=\frac{1}{2}\left(W_{V}+W_{0}\right)+\mathrm{i} \operatorname{Im} W
$$

with

$$
\operatorname{Im} W \equiv \operatorname{Im} W(a), a_{\mu}=\frac{2 \pi}{T_{\mu}} t_{\mu}
$$

being given by $(2 \cdot 30)$. The result for the real part, namely that it is given by half the 
action of the vector theory, was already conjectured in ref. 13. The imaginary part of the effective action can be computed analytically directly from the lattice gauge field. Thus we have arrived at an action which can be simulated on the original lattice with not much more effort than that of the vector theory.

In the sector of non-trivial topology the result for the real part has to be modified, while the result for the imaginary part will be unchanged. We shall return to this problem in the near future.

We see no problem in extending the method to higher dimensions. Work on a chiral $\mathrm{U}(1)$ model in four dimensions is in progress.

\section{Acknowledgement}

One of us (G.S.) thanks M. Göckeler for discussions on the subject of this talk. He also likes to thank T. Suzuki for his kind hospitality at Kyoto as well as JSPS for financial support.

\section{References}

1) M. Göckeler and G. Schierholz, Nucl. Phys. B (Proc. Suppl.) 29B,C (1992) 114; ibid. 30 (1992) 609; G. 't Hooft, Phys. Lett. B349 (1995) 491.

2) M. Göckeler, A. S. Kronfeld, G. Schierholz and U.-J. Wiese, Nucl. Phys. B404 (1993) 839.

3) P. Hernandez and R. Sundrum, Nucl. Phys. B455 (1995) 287; G. T. Bodwin, Phys. Rev. D54 (1996); I. Montvay, CERN-TH-95-123 (1995) (hep-lat/9505015).

4) J. Schwinger, Phys. Rev. 128 (1962) 2425.

5) C. Jayewardena, Helv. Phys. Acta 61 (1988) 636.

6) H. Joos, Helv. Phys. Acta 63 (1990) 670; H. Joos and S. I. Azakov, ibid. 67 (1994) 723.

7) I. Sachs and A. Wipf, Helv. Phys. Acta 65 (1992) 652.

8) S. Azakov, Fortschr. Phys. 45 (1997) 589.

9) R. Jackiw and R. Rajamaran, Phys. Rev. Lett. 54 (1985) 1219.

10) S. A. Dias and C. A. Linhares, Phys. Rev. D45 (1992) 2162.

11) R. Jackiw in Current algebra and anomalies, eds. S.B. Treiman, R. Jackiw, B. Zumino and E. Witten (World Scientific 1985).

12) S. A. Dias and C. A. Linhares, Phys. Rev. D47 (1993) 1672.

13) L. Alvarez-Gaumé and S. Della Pietra, in Recent Developments in Quantum Field Theory, eds. J. Ambjorn, B. J. Durhuus and J. L. Petersen (North-Holland, 1985); L. AlvarezGaumé, S. Della Pietra and V. Della Pietra, Phys. Lett. B166 (1986) 177.

14) L. Alvarez-Gaumé, G. Moore and C. Vafa, Commun. Math. Phys. 106 (1986) 1; M. Göckeler and G. Schierholz, unpublished (1994); R. Narayanan and H. Neuberger, Phys. Lett. B348 (1995) 549.

15) M. F. L. Golterman and D. N. Petcher, Phys. Lett. B225 (1989) 159.

16) A. Borrelli, L. Maiani, G. C. Rossi, R. Sisto and M. Testa, Nucl. Phys. B333 (1990) 335.

17) V. Bornyakov, A. Thimm and G. Schierholz, in preparation.

18) I. M. Barbour, N.-E. Behilil, P. E. Gibbs, G. Schierholz and M. Teper, in Springer Series in Solid State Sciences 58, The Recursion Method and its Applications, p. 149, eds. D. Pettifor and D. L. Weaire (Springer,1985).

19) V. Bornyakov, A. Thimm and G. Schierholz, Nucl. Phys. B (Proc. Suppl.) 63 (1998) 593; DESY 98-043 (1998) (hep-lat/9805007). 